\title{
Optimisation of Discrete Processes with Bounded Control
}

\section{Optimisation of \\ Discrete Processes with Bounded Control}

\section{Marek Milosz}

Institute of Computer Science; Lublin University of Technology;

Nadbystrzycka Str. 36B, 20-618, Lublin, Poland; e mail: m.milosz@pollub.pl

\section{Zainel Murzabekov, Kamshat Tussupova}

Department of Information Systems; Al-Farabi Kazakh National University; ave. al-Farabi 71, 050040, Almaty, Kazakhstan; e-mails: murzabekov-zein@mail.ru, kamshat-0707@mail.ru

\section{Saltanat Usubalieva}

Narxoz University; Dzhandosov Str. 55, 050035, Almaty, Kazakhstan; e-mail: saltanat.usubalieva@narxoz.kz

\section{Corresponding author:m.milosz@pollub.pl}

The study considers the problem of optimal control for linear discrete systems with a free right end of the trajectory and constraints on control. A new approach to constructing a discrete system is proposed and control is determined at discrete instants of time. Necessary and sufficient conditions for optimality are obtained and a method is proposed for the exact solution of the boundary value problem, which is reduced to solving a finite number of systems of algebraic equations. The proposed method for solving the optimal control problem for a discrete system allows to represent the desired optimal control in the form of synthesising control. For this, a positively definite symmetric matrix is defined that satisfies a difference equation of Riccati type. An algorithm for constructing control for discrete systems is developed, based on the feedback principle, taking into account constraints on the values of controls. The problem is solved using the Lagrange multipliers of a special form, which depend on the phase coordinates at discrete instants of time. The proposed method for solving the problem of optimal control with constraints on control values is implemented on a computer with an application package and tested for the task of planning production and storage of products. Numerical calculations are carried out on a computer using the described problem-solving algorithm in which it is possible to take into account restrictions on the values of controls. Optimal values are determined and appropriate schedules of the production plan, storage of products and limited management at discrete instants of time are constructed.

KEYWORDS: optimal control problem, Lagrange multiplier method, discrete systems, quadratic functional, production and storage of products. 


\section{Introduction}

Development of models of various discrete processes and control algorithms with the necessary properties for their application, taking into account their limited discrete control, are becoming increasingly important for the theory and practice of optimal control and modern information technologies. This is connected with the fact that many of the tasks of economic planning are described by difference equations.

Real information about the process often arrives at discrete points in time, and the process control should be carried out at discrete times. The tasks of this kind are both long-term and operational planning, programming development of various systems, the problems of production and storage planning, planning multi-process systems.

Historically, the discrete systems optimal control theory was developed after the continuous systems control theory. The basis for methods of continuous optimal control with the construction of program management is the Pontryagin's maximum principle [6, 13, 16]. The solution to the same problem is feedback [15] optimal control synthesis based on Bellman's method of dynamic programming [5]. Optimality conditions in the form of the maximum principle for continuous systems are the most versatile and practical method. Therefore, it is not surprising that the maximum principle ideas also have a strong influence on the theory of discrete optimal control $[2,3,11,14]$.

The problem of optimal control for discrete systems can be formulated as the problem of finding openloop (programmed) control or as a task of constructing closed-loop (synthesis) control. In the first case, the problem can be solved using the Pontryagin maximum principle. However, the fact that this principle is only a necessary condition for optimality limits the possibilities of its application. In addition, there arises a two-point boundary value problem for ordinary differential equations, the solution of which is associated with some computational difficulties, especially when there are constraints on the control parameters. In the second case, Bellman's dynamic programming method can be used to solve the problem. In this method, difficulties arise associated with the choice of the corresponding Bellman functions satisfying certain boundary conditions. However, it should be noted that this method has not been sufficiently stud- ied for discrete dynamical systems in the presence of external influences, and especially with limited values of the controls.

The problem of studying stability and the synthesis of stabilizing control has been given considerable attention in the literature in connection with its relevance. The paper [9] formulated and discussed local problems of limited controllability of discrete control systems with constant coefficients.

Applications of discrete control are associated mainly with economical problems, organisation and technology of production, and operational research. Such problems are characterised by large numbers of both state and control variables and constraints, this being the reason of a close relationship of these problems to mathematical programming $[4,7,10]$.

In this paper we consider a discrete linear system with limited control and offer a new approach to modelling and design of discrete systems, as well as the appropriate control algorithm based on the feedback principle with constraints on the values of the control.

\section{Problem of Discrete Control}

Let us consider a discrete control problem in which the process is described by a linear difference equation:

$$
\begin{aligned}
& x(k+1)=A(k) x(k)+B(k+1) u(k+1)+f(k+1), \\
& k \in T=\{0,1, \ldots, N-1\}, \quad x(0)=x_{0}, \\
& \hline u(k+1) \in U(k+1)=\{u(k+1) \mid \\
& \alpha(k+1) \leq u(k+1) \leq \beta(k+1), \quad k \in T\},
\end{aligned}
$$

where $x(k) \in R^{n}$ is a state vector; $u(k) \in U(k) \subseteq R^{m}$ is a vector of control; $f(k) \in R^{n}$ is a given vector; $k$ is a discrete time moment; $N$ is a specified number of steps.

It is assumed that for determination of control information about the current time $k$ and the current state vector $x(k)$ is used. Discrete control is regarded as a multi-step process. At each $k$-th step, the process is characterised by two variables $x(k)$ and $u(k)$. Vector $x(k)$, which specifies the state of the process at the $k$-th step, is an element of the $n$-dimensional real Euclidean space $R^{n}$. Vector $u(k)$ determines the control action at the $k$-th step and belongs to a given 
subset $U(k)$ of the Euclidean space $R^{m}$ (the space of control actions).

Let a cost-functional be given which depends on both the control and state of the object:

$$
\begin{aligned}
& J(x(k), u(k+1))=\sum_{k=0}^{N-1}\left[\frac{1}{2} x^{*}(k) Q(k) x(k)+c^{*}(k) x(k)\right. \\
& \left.+d^{*}(k+1) u(k+1)+\frac{1}{2} u^{*}(k+1) R(k+1) u(k+1)\right] \\
& +\frac{1}{2} x^{*}(N) F x(N)+c^{*}(N) x(N)
\end{aligned}
$$

Here $Q(k)$ is a positive semi-definite symmetric matrix, and $R(k), F$ are positive definite symmetric matrices.

The task is to find a synthesising control $u(x(k), k)$ such that the corresponding pair $(x(k), u(k))$ gives the minimum of functional (3) and satisfies Equation (1) under constraints (2) on the control.

To solve the linear-quadratic problem (1)-(3) we use a method based on the special type of Lagrange multipliers $[1,12]$. We add to the expression for functional (3) a system of difference Equations (1) with the multiplier $\frac{1}{2} P(k+1) x(k+1)+q(k+1)$, as well as the following item:

$\lambda_{1}^{*}(k+1)[\alpha(k+1)-u(k+1)]+\lambda_{2}^{*}(k+1)[u(k+1$

$-\beta(k+1)]+\lambda_{3}^{*}(k)[x(k)-W(k) q(k)-z(k)]$,

where $\lambda_{1}(k+1) \geq 0, \lambda_{2}(k+1) \geq 0$. As a result, we obtain the following functional:

$$
\begin{aligned}
& L(x(k), u(k+1))=\frac{1}{2} x^{*}(N) F x(N)+c^{*}(N) x(N) \\
& +\sum_{k=0}^{N-1}\left[\frac{1}{2} x^{*}(k) Q(k) x(k)+c^{*}(k) x(k)+d^{*}(k+1)\right. \\
& \left.\times u(k+1)+\frac{1}{2} u^{*}(k+1) R(k+1) u(k+1)\right] \\
& +\left[\frac{1}{2} P(k+1) x(k+1)+q(k+1)\right]^{*}[A(k) x(k) \\
& +B(k+1) u(k+1)+f(k+1)-x(k+1)] \\
& +\lambda_{1}^{*}(k+1)[\alpha(k+1)-u(k+1)]+\lambda_{2}^{*}(k+1)[u(k+1) \\
& -\beta(k+1)]+\lambda_{3}^{*}(k+1)[x(k)-W(k) q(k)-z(k)],
\end{aligned}
$$

where $q(k)$ is an $n$-vector, and $P(k)$ is an $(n \times n)$-matrix. The multiplier $\frac{1}{2} P(k+1) x(k+1)+q(k+1)$ eliminates the constraints on the admissible pairs $(x(k), u(k))$ in the form of a system of difference Equations (1), and multipliers $\lambda_{1}(k), \lambda_{2}(k)$ eliminate constraints on the control in (2); multiplier $\lambda_{3}(k)$ retains the properties of the system to satisfy the boundary conditions. Such design of functional (4) allows to convert the original conditional extremum problem to an unconditional extremum one.

Using the following expressions:

$\frac{1}{2} \sum_{k=0}^{N-1}\left[x^{*}(k) P(k) x(k)-x^{*}(k+1) P(k+1) x(k+1)\right]$
$=\frac{1}{2}\left[x^{*}(0) P(0) x(0)-x^{*}(N) P(N) x(N)\right]$,
$\sum_{k=0}^{N-1}\left[q^{*}(k) x(k)-q^{*}(k+1) x(k+1)\right]=q^{*}(0) x(0)-q^{*}(N) x(N)$,

we convert the functional (4) to the form:

$L(x(k), u(k+1))=F_{1}(x(N), x(0))$

$+\sum_{k=0}^{N-1} M(x(k), u(k+1))$.

The following notations are used:

$F_{1}(x(N), x(0))=\frac{1}{2} x^{*}(N)[F-P(N)] x(N)+c^{*}(N) x(N)$

$-q^{*}(N) x(N)+\frac{1}{2} x^{*}(0) P(0) x(0)+q^{*}(0) x(0)$,

$M(x(k), u(k+1))=\frac{1}{2} x^{*}(k)[Q(k)-P(k)] x(k)$

$\left.+\frac{1}{2} u^{*}(k+1) R(k+1) u(k+1)\right]+c^{*}(k) x(k)$

$+d^{*}(k+1) u(k+1)+\left[\frac{1}{2} P(k+1)(A(k) x(k)\right.$

$+B(k+1) u(k+1)+f(k+1)$

$+q(k+1)]^{*}[A(k) x(k)+B(k+1) u(k+1)+f(k+1)]$

$-q^{*}(k) x(k)+\lambda_{1}^{*}(k+1)\left[\alpha(k+1)-u(k+1]+\lambda_{2}^{*}(k+1)\right.$

$\times[u(k+1)-\beta(k+1)]+\lambda_{3}^{*}(k)[x(k)-W(k) q(k)-z(k)]$.

Now we choose control $u(k+1)$ such that the function $L(x(k), u(k+1))$ reaches a minimum by $u(k+1)$ and $x(k)$ at any $k \in T$. The necessary conditions for a minimum of the functional (5) are the following:

$$
\begin{aligned}
& \frac{\partial M(x(k), u(k+1))}{\partial u(k+1)}=R(k+1) u(k+1)+d(k+1) \\
& +B^{*}(k+1)\{P(k+1)[A(k) x(k)+B(k+1) u(k+1) \\
& +f(k+1)]+q(k+1)\}-\lambda_{1}(k+1)+\lambda_{2}(k+1)=0,
\end{aligned}
$$




$$
\begin{aligned}
& \frac{\partial M(x(k), u(k+1))}{\partial x(k)}=[Q(k)-P(k)] x(k)+c(k) \\
& +A^{*}(k) P(k+1)[A(k) x(k)+B(k+1) u(k+1) \\
& +f(k+1)]+A^{*}(k) q(k+1)-q(k)+\lambda_{3}(k)=0, \\
& \frac{\partial F_{1}(x(N), x(0))}{\partial x(N)}=[F-P(N)] x(N) \\
& +c(N)-q(N)=0 .
\end{aligned}
$$

Now we find, using (6)

$$
\begin{aligned}
& u(x(k), k+1)=-\left[R(k+1)+B^{*}(k+1)\right. \\
& \times P(k+1) B(k+1)]^{-1}\left[B^{*}(k+1) P(k+1) A(k) x(k)\right. \\
& +B^{*}(k+1) q(k+1)+d(k+1)+B^{*}(k+1) P(k+1) \\
& \left.\times f(k+1)-\lambda_{1}(k+1)+\lambda_{2}(k+1)\right] .
\end{aligned}
$$

To fulfill conditions (6)-(8), $P(k)$ is used, a positive definite symmetric matrix, which satisfies the equation:

$$
\begin{aligned}
& P(k)=Q(k)+A^{*}(k) P(k+1) A(k)-A^{*}(k) P(k+1) \\
& \times B(k+1)\left[R(k+1)+B^{*}(k+1) P(k+1) B(k+1)\right]^{-1} \\
& \times B^{*}(k+1) P(k+1) A(k), \quad P(N)=P_{N},
\end{aligned}
$$

and vector $q(k)$ is determined from the equation:

$$
\begin{aligned}
& q(k)=A_{1}^{*}(k) q(k+1)+c(k)-\left[A_{1}(k) W(k)\right]^{-1} \\
& \times B(k+1) \varphi(k+1)+A^{*}(k) P(k+1) f(k+1) \\
& -A^{*}(k) P(k+1) B(k+1)\left[R(k+1)+B^{*}(k+1)\right. \\
& \times P(k+1) B(k+1)]^{-1}\left[B^{*}(k+1) P(k+1) f(k+1)\right. \\
& +d(k+1)], \quad q(N)=[F-P(N)] x(N)+c(N) .
\end{aligned}
$$

The multiplier $\lambda_{3}(k)$ and matrix $W(k)$ are defined as follows:

$$
\begin{aligned}
& \lambda_{3}(k)=-\left[A^{*}(k) P(k+1) B(k+1)\right. \\
& \left.+\left(A_{1}(k) W(k)\right)^{-1} B(k+1)\right] \varphi(k+1), \\
& \hline W(k+1)=A_{1}(k) W(k) A_{1}^{*}(k)-B_{1}(k+1), \\
& W(N)=(F-P(N))^{-1} . \\
& \text { The following notations are used in Equ } \\
& A_{1}(k)=A(k)-B(k+1)\left[R(k+1)+B^{*}(k+1)\right. \\
& \times P(k+1) B(k+1)]^{-1}\left[B^{*}(k+1) P(k+1) A(k)\right], \\
& B_{1}(k+1)=B(k+1)\left[R(k+1)+B^{*}(k+1)\right. \\
& \times P(k+1) B(k+1)]^{-1} B^{*}(k+1),
\end{aligned}
$$

The following notations are used in Equations (11)-(13):

$$
\begin{aligned}
& \varphi_{1}(k+1)=\left[E-R_{1}^{-1}(k+1) B^{*}(k+1)\left(A_{1}^{*}(k)\right)^{-1}\right. \\
& \left.\times W^{-1}(k)\left(A_{1}(k)\right)^{-1} B(k+1)\right] \varphi(k+1),
\end{aligned}
$$

$$
\begin{aligned}
& \varphi(k+1)=-\left(R_{1}(k+1)\right)^{-1}\left[-\lambda_{1}(k+1)+\lambda_{2}(k+1)\right], \\
& R_{1}(k+1)=R(k+1)+B^{*}(k+1) P(k+1) B(k+1) .
\end{aligned}
$$

Suppose that there exists a solution of Equations (10)-(13), then conditions (6)-(8) are fulfilled, and the equation that determines the law of motion of system (1) with control (9) is as follows:

$$
\begin{aligned}
& x(k+1)=A_{1}(k) x(k)-B_{1}(k+1) q(k+1) \\
& -B(k+1) R_{1}^{-1}(k+1) d(k+1)+B(k+1) \varphi(k+1) \\
& +\left[E-B_{1}(k+1) P(k+1)\right] f(k+1), \quad x(0)=x_{0} .
\end{aligned}
$$

Note that for the determination of the initial condition $q(0)=q_{0}$ for Equation (11), the following relation is expedient:

$x(k)=W(k) q(k)+z(k)$,

where the matrix $W(k)$ satisfies Equation (13), and function $z(k)$ is determined from the solution of the vector equation:

$$
\begin{aligned}
& z(k+1)=A_{1}(k) z(k)+\left[E+A_{1}(k) W(k) A^{*}(k)\right. \\
& \times P(k+1)]\left[E-B_{1}(k+1) P(k+1)\right] f(k+1) \\
& +A_{1}(k) W(k) c(k)-\left[E+A_{1}(k) W(k) A^{*}(k)\right. \\
& \times P(k+1)] B(k+1)\left(R_{1}(k+1)\right)^{-1} d(k+1), \\
& z(N)=-[F-P(N)]^{-1} c(N) .
\end{aligned}
$$

Hence, using the solutions of Equations (13) and (16), we find from (15) the initial condition for Equation (11):

$$
q(0)=q_{0}=W^{-1}(0)[x(0)-z(0)]
$$

To determine multipliers $\lambda_{1}(k+1), \lambda_{2}(k+1)$, we introduce the notations:

$$
\begin{aligned}
& w(x(k), k+1)=-R_{1}^{-1}(k+1)\left[B^{*}(k+1) P(k+1)\right. \\
\times & A(k) x(k)+B^{*}(k+1)\left(A_{1}^{*}(k)\right)^{-1} q(k) \\
+ & {\left[E+B^{*}(k+1)\left(A_{1}^{*}(k)\right]^{-1} A^{*}(k) P(k+1) B(k+1)\right.} \\
\times & \left.\left.R_{1}^{-1}(k+1)\right) d(k+1)-B^{*}(k+1)\left(A_{1}^{*}(k)\right)^{-1} c(k)\right], \\
& R_{2}(k+1)=\left[E-R_{1}^{-1}(k+1) B^{*}(k+1)\left(A_{1}^{*}(k)\right)^{-1}\right. \\
\times & \left.W^{-1}(k) A_{1}^{-1}(k) B(k+1)\right] R_{1}^{-1}(k+1) .
\end{aligned}
$$

The multipliers $\lambda_{1}(k+1) \geq 0, \lambda_{2}(k+1) \geq 0$, and control $u(k+1)$ will be defined so that the following conditions hold: 


$$
\begin{aligned}
& \lambda_{1}^{*}(k+1)[\alpha(k+1)-u(k+1)]=0, \\
& \lambda_{2}^{*}(k+1)[u(k+1)-\beta(k+1)]=0, \\
& u(x(k), k+1)-w(x(k), k+1)] \\
& +R_{2}(k+1)\left[-\lambda_{1}(k+1)+\lambda_{2}(k+1)\right]=0 .
\end{aligned}
$$

To do this, we use the following expressions:

$$
\begin{aligned}
& \lambda_{1}^{0}(k+1)=-R_{2}^{-1}(k+1) \min \{0, w(x(k), k+1)-\alpha(k+1)\} \\
& \lambda_{2}^{0}(k+1)=-R_{2}^{-1}(k+1) \min \{0, \beta(k+1)-w(x(k), k+1)\} .
\end{aligned}
$$

Using the results (19), we choose $\lambda_{1}(k+1), \lambda_{2}(k+1)$ and $u(k+1)$ as follows:

_ if $\lambda_{1 i}^{0}(k+1)=0, \lambda_{2 i}^{0}(k+1)=0$, then take $\lambda_{1 i}(k+1)=0$, $\lambda_{2 i}(k+1)=0$, and determine $u_{i}(k+1)$ from (18);

_ if $\lambda_{1 i}^{0}(k+1)>0$, then take $\lambda_{2 i}(k+1)=0, u_{i}(k+1)=$ $\alpha_{i}(k+1)$, and determine $\lambda_{1 i}(k+1)$ from (18);

- if $\lambda_{2 i}^{0}(k+1)>0$, then take $\lambda_{1 i}(k+1)=0, u_{i}(k+1)=$ $\beta_{i}(k+1)$, and determine $\lambda_{2 i}(k+1)$ from (18).

Thus, we get the following result for the problem in question:

Theorem: The pair $(\tilde{x}(k), \tilde{u}(k+1))$ in problem (1)-(3) is optimal if and only if the state vector $x(k)$ satisfies the difference equation:

$$
\begin{aligned}
& x(k+1)=A_{1}(k) x(k)-B_{1}(k+1)\left(A_{1}^{*}(k)\right)^{-1} q(k) \\
& +B_{1}(k+1)\left(A_{1}^{*}(k)^{-1} c(k)-\left[B_{1}(k+1)\left(A_{1}^{*}(k)\right)^{-1}\right.\right. \\
& \left.\times A^{*}(k) P(k+1)+E\right] B(k+1) R_{1}^{-1}(k+1) d(k+1) \\
& +\left(E-B_{1}(k+1)\left(A_{1}^{*}(k)\right)^{-1} W^{-1}(k) A_{1}^{-1}(k)\right. \\
& \times B(k+1) \varphi(k+1)+f(k+1), x(0)=x_{0} .
\end{aligned}
$$

Vector $q(k)$ is determined from the difference equation: $q(k+1)=\left(A_{1}^{*}(k)\right)^{-1}\left[q(k)-c(k)+A^{*}(k) P(k+1)\right.$

$\times B(k+1) R_{1}^{-1}(k+1) d(k+1)+W^{-1}(k) A_{1}^{-1}(k)$

$\times B(k+1) \varphi(k+1)\}-P(k+1) f(k+1), \quad q(0)=q_{0}$.

Control $u(x(k), k+1)$ is determined as follows:

$$
\begin{aligned}
& u(x(k), k+1)=-R_{1}^{-1}(k+1)\left\{B^{*}(k+1)\right. \\
& \times P(k+1) A(k) x(k)+B^{*}(k+1)\left(A_{1}^{*}(k)\right)^{-1} q(k) \\
& +\left[E+B^{*}(k+1)\left(A_{1}^{*}(k)\right)^{-1} A^{*}(k) P(k+1)\right. \\
& \left.\times B(k+1) R_{1}^{-1}(k+1)\right] d(k+1) \\
& \left.-B^{*}(k+1)\left(A_{1}^{*}(k)\right)^{-1} c(k)\right\}+\varphi_{1}(k+1),
\end{aligned}
$$

where matrix $P(k+1)$ is determined from the difference Equation (10), and function $\varphi(k+1)$ from (14).

The algorithm for solving the problem. We offer here the algorithm for solving the optimal control problem (1)-(3), convenient to implement on a computer:

1 Determine the positive definite symmetric matrices $P(k)$ for $k \in T=\{0,1, \ldots, N-1\}$, using a system of difference Equations (10).

2 Determine matrices $W(k)$ for $k \in T$, using a system of difference Equations (13).

3 Determine function $z(k)$ as the solution of the vector Equation (16).

4 Define conditions $x(0)=x_{0}, z(0)$, and calculate initial conditions $q(0)$ for the difference Equation (11) in accordance with (17).

5 Determine control $(q(k+1), x(k+1))$ for $k \in T$, with the initial conditions $q(0)=q_{0}$ and $x(0)=x_{0}$, using the system of difference Equations (11), (20).

In the process of solving the difference equations, the values of optimal control $u(x(k), k+1$ are calculated as well, according to Formula (21).

\section{An Example: Production and Storage of Produce}

It is necessary to develop an output schedule of seasonal ingredients. The entrepreneur should plan the monthly output of the product for the next year. Estimated demand for products during the year is presented in Table 1.

The entrepreneur is obliged to meet the monthly requirements defined by this schedule. It is necessary to determine the output schedule, which minimises the deviation from the average demand and stock of goods, arising from the production, and storage fluctuations.

The entrepreneur, faced with fluctuations in demand, can produce the additional product in a low demand period, in order to store and use it in the period of excess demand.

The production process can then be made very stable. However, due to increased costs associated with the storage of surplus, this solution may be unacceptable if it leads to large monthly surpluses. Problems of this 
Table 1

Estimated demand for products

\begin{tabular}{c|c}
\hline Month, $k$ & Demand, $r_{k}$ \\
\hline 1 & 30 \\
\hline 2 & 60 \\
\hline 3 & 40 \\
\hline 4 & 70 \\
\hline 5 & 90 \\
\hline 6 & 110 \\
\hline 7 & 90 \\
\hline 8 & 90 \\
\hline 9 & 60 \\
\hline 10 & 80 \\
\hline 11 & 90 \\
\hline 12 & 120 \\
\hline
\end{tabular}

kind illustrate the difficulties that arise in the presence of factors affecting adversely the cost function [8].

The corresponding mathematical model for the problem of production and storage planning is now represented in the following form:

$\min z=\frac{1}{2} \sum_{k=0}^{N-1}[s(k)-50]^{2}+\frac{5}{2} \sum_{k=0}^{N-1}[x(k)-75]^{2}$

$+\frac{4}{2} \sum_{k=0}^{N-1}[u(k+1)-20]^{2}+\frac{10}{2}[s(N)-1]^{2}+\frac{10}{2}[x(N)-75]^{2}$

by the conditions:

$\left\{\begin{array}{l}s(k+1)=s(k)+x(k)+u(k+1)-r(k+1), \\ x(k+1)=x(k)+u(k+1),\end{array}\right.$

$x(k+1) \geq 0, \quad s(k+1) \geq 0, \quad \alpha \leq u(k+1) \leq \beta$,

$k \in T=\{0,1, \ldots, N-1\}, \quad N=12, \quad \alpha=-5, \quad \beta=15$,

where

$$
\begin{aligned}
& A(k)=\left(\begin{array}{ll}
1 & 1 \\
0 & 1
\end{array}\right), \quad b(k+1)=\left(\begin{array}{l}
1 \\
1
\end{array}\right), \quad f(k+1)=\left(\begin{array}{c}
-r_{k+1} \\
0
\end{array}\right), \\
& Q(k)=\left(\begin{array}{ll}
1 & 0 \\
0 & 5
\end{array}\right), \quad c(k)=\left(\begin{array}{c}
-50 \\
-375
\end{array}\right), \quad d(k+1)=-80, \\
& R(k+1)=4, \quad F=\left(\begin{array}{rr}
10 & 0 \\
0 & 10
\end{array}\right) .
\end{aligned}
$$

To solve this problem, we used an algorithm for optimal control problems proposed in Section 2. The values $(s(k+1), x(k+1))$ for $k \in T=\{0,1, \ldots, N-1\}$ with the initial conditions $(s(0)=30, x(0)=30)$ are determined using a system of difference Equations (20). The desirable optimal control is written in the form (21). Graphs of the optimal output, stock and control are represented in Figures 1-3.

\section{Figure 1}

Graph of the optimal trajectory of production output

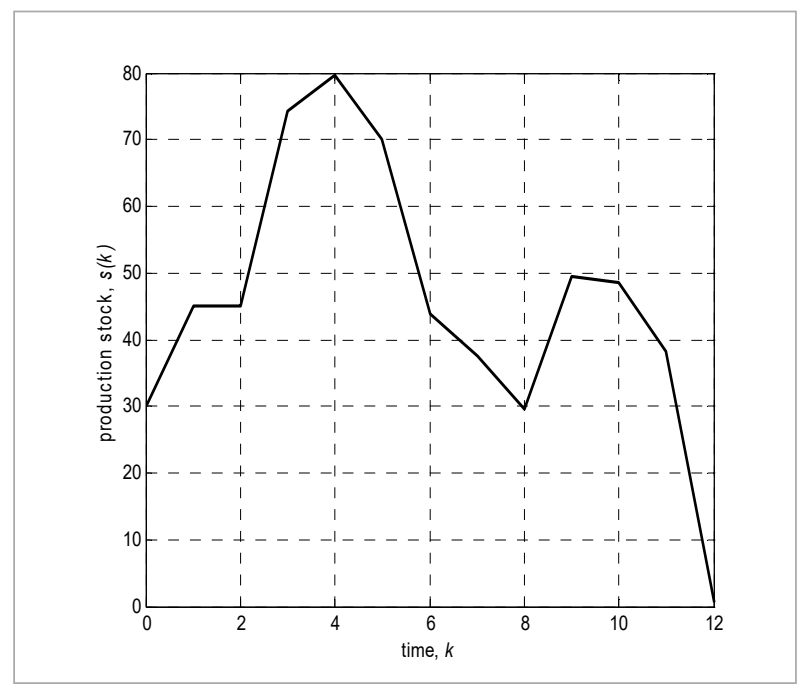

Figure 2

Graph of the optimal trajectory of production stock

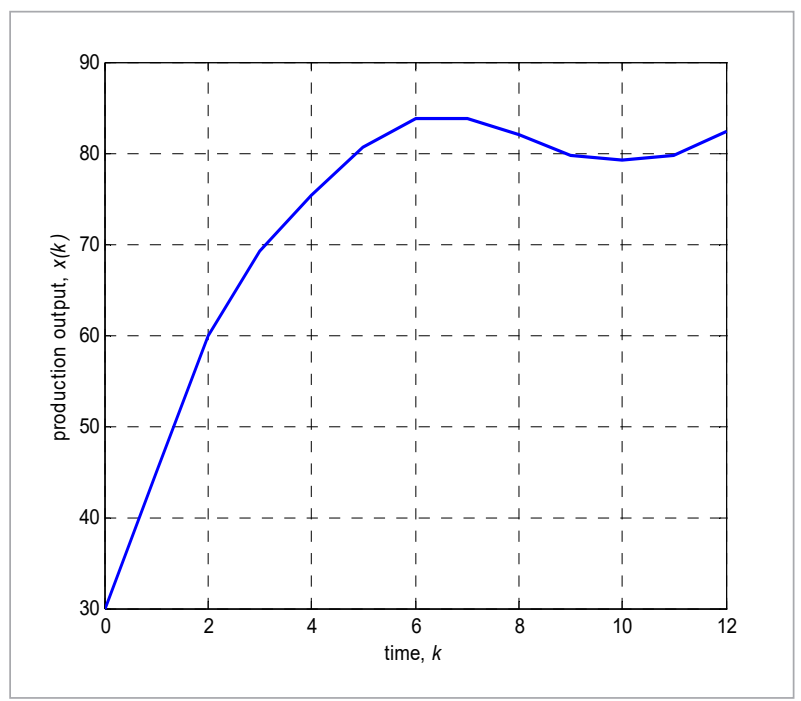


Figure 3

Graph of the optimal control

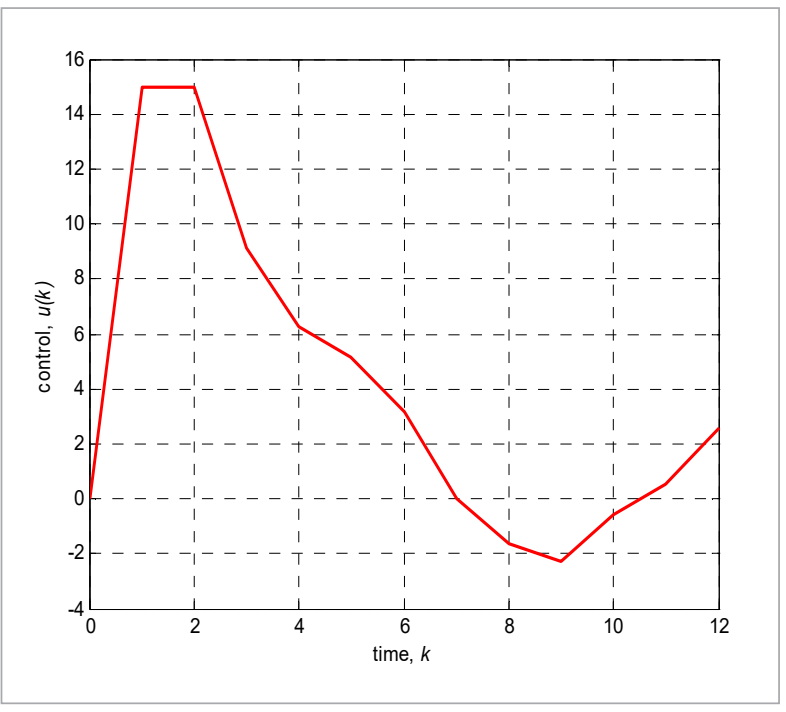

\section{References}

1. Aipanov, Sh. A., Murzabekov, Z. N. Analytical Solution of a Linear Quadratic Optimal Control Problem with Control Value Constraints. Journal of Computer and Systems Sciences International, 2014, 53(1), 84-91. https://doi.org/10.1134/S1064230713060026

2. Alekseev, V. M., Tikhomirov, V. M., Fomin, S. V. Optimal Control. Consultants Bureau, NY, 1987. https://doi. org/10.1007/978-1-4615-7551-1

3. Athans, M., Falb, P. L. Optimal Control: An Introduction to the Theory and Its Applications. Dover Publications, Inc., Mineola, NY, 2007.

4. Backhoff, J., Silva, F. J. Sensitivity Results in Stochastic Optimal Control: A Lagrangian Perspective. ESAIM Control, Optimisation and Calculus of Variations, 2017, 23(1), 39-70. https://doi.org/10.1051/cocv/2015039

5. Bellman, R. Dynamic Programming. Princeton University Press, Princeton, 2010.

6. Boltyanskii, V. G. Optimal Control of Discrete Systems. John Wiley \& Sons Inc., NY, 1978.

7. Farokhi, F., Johansson, K. Optimal Control Design Under Limited Model Information for Discrete-Time Linear Systems with Stochastically-Varying Parameters. IEEE Transactions on Automatic Control, 2015, 60(3), 684-699. https://doi.org/10.1109/TAC.2014.2343091

8. Gass, S. I. Linear Programming: Methods and Applications. 5th ed.; Publisher: Dover Publications, Mineola, NY, 2003.

9. Klamka, J. Controllability of Nonlinear Discrete Systems. International Journal of Applied Mathematics

\section{Conclusions}

In this paper, we propose a new approach to designing control and the corresponding control algorithm, based on the feedback principle, for discrete systems with constraints on the values of the control. The problem is solved using Lagrange multipliers of a special type, depending on the phase coordinates at discrete time points.

The proposed method and algorithm are tested for the problem of production planning. The optimal values of output, stocks and control with proper constraints are determined and corresponding graphs are constructed at discrete time points.

and Computer Science, 2002, 12(2), 173-180. https:// doi.org/10.1109/ACC.2002.1025394

10. Lanćinskas, A., Fernandez, P., Pelegrin, B., Zilinskas, J. Solution of Discrete Competitive Facility Location Problem for Firm Expansion. Informatica, 2016, 27(2), 451-462. https://doi.org/10.15388/Informatica.2016.94

11. Luo, B., Liu, DR., Huang, T. W., Yang, X., Ma, H. W. Multi-Steps Heuristic Dynamic Programming for Optimal Control of Nonlinear Discrete-Time Systems. Information Sciences, 2017, 411, 66-83. https://doi.org/10.1016/j.ins.2017.05.005

12. Murzabekov, Z. N. Dostatochnye Usloviya Optimal'nosti Dinamicheskikh Sistem Upravleniya s Zakreplennymi Kontsami. Matematicheskii Zhurnal, 2004, 2 (4), 52-59 (in Russian).

13. Pontryagin, L. S., Boltyanskii, V.G., Gamkrelidze, R.V., Mishchenko, E.F. The Mathematical Theory of Optimal Processes. L. S. Pontryagin Selected Works, 4, Gordon \& Breach Science Publishers, NY, 1986.

14. Propoi, A. I. Elementy Teorii Optimal'nykh Diskretnykh Protsessov. Nauka, Moskva, 1973 (in Russian).

15. Qiu, J., Wei, Y., Karimi, H. R., Gao, H. Reliable Control of Discrete-Time Piecewise-Affine Time-Delay Systems via Output Feedback. IEEE Transactions on Reliability, 2017, 67(1), 79-91. https://doi.org/10.1109/ TR.2017.2749242

16. Ross, I. M. A Primer on Pontryagin's Principle in Optimal Control. 2nd ed., Collegiate Publishers, San Francisco, 2015. 